\title{
Das globale Unten: Die Konstruktion eines globalen medizinischen Südens in den USA
}

Tine Hanrieder

\section{Abstract}

Der Beitrag diskutiert Vorstellungen von Globalität in Theorie und Praxis der Global Governance. Er stellt der Idee einer globalen Gemeinschaft, wie sie in Theorien der Global Governance und der Internationalen Beziehungen (IB) vorherrscht, eine sozialgeographische Sicht gegenüber, die die Konstruktion postnationaler Raumhierarchien herausarbeitet. Am Beispiel der Konstruktion von sogenannten globalen Orten in der US Medizin zeige ich, wie auf diskursiver, institutioneller und politikprogrammatischer Ebene heimische Peripherien in einen globalen medizinischen Süden eingemeindet werden. Der Rückgriff aufs Globale normalisiert in diesem Kontext Ungleichheiten im eigenen Land, die durch räumliche Konnotationen aus der Entwicklungspolitik noch natürlicher erscheinen.

\section{Einleitung}

Die Begeisterung für Global Governance ist ungleich verteilt, nicht nur zwischen, sondern auch innerhalb von Ländern. ${ }^{1}$ Dies zeigen nicht zuletzt Michael Zürns Arbeiten zur Umstrittenheit globaler Institutionen (Zürn 2018) und zu neuen gesellschaftlichen cleavages, die sich zwischen elitären Globalisierungsbefürworterinnen und abhängten Globalisierungskritikerinnen auftun (de Wilde et al. 2019).

Im Lichte dieser Beobachtungen diskutiert der vorliegende Beitrag, ob ein wenig hinterfragter Kernbegriff der Global Governance - nämlich Globalität - den beobachteten transnationalen Dynamiken und Verwerfungen angemessen ist. Globalität wird in der Literatur zu Global Governance und auch in Zürns Arbeiten im Sinne von Supraterritorialität gedeutet - als der ortlose Ort globaler Institutionen und universeller Werte (Zürn 2013; Zürn 2018: 25-36, und siehe unten, Abschnitt 2). Ich schlage demgegenüber vor, einen sozialgeographischen Blick auf Global Governance zu werfen, um die Ordnungsvorstellungen von Global Governance anhand

\footnotetext{
${ }^{1}$ Ich danke meinem Kommentator Pieter de Wilde und allen weiteren Teilnehmerinnen und Teilnehmern des Workshops zur Zukunft der Global Governance für ihre konstruktiven Kommentare, ebenso den beiden anonymen Gutachter/innen.
} 
ihrer Verräumlichung zu erfassen. Die geographische Perspektive ermöglicht es, etablierte Vorstellungen des Globalen als supraterritorialen Raum geteilter Werte zu überschreiten, und stattdessen räumlich konnotierte Hierarchien und damit einhergehende symbolische Grenzziehungen zu betrachten, die mit postnationaler Entgrenzung einhergehen.

Konkret untersuche ich die Konstruktion von Globalität am Beispiel Weltgesundheitspolitik Global Health Governance.Dieses Politikfeld steht exemplarisch für weit verbreitete Vorstellungen von Globalität in der Global Governance. Transnationale Verflechtungen und beschleunigte Reise- und Transportbewegungen sorgen seit Langem dafür, dass beispielsweise Infektionskrankheiten nicht an Grenzen Halt machen - das heißt für eine Kompression von Raum und Zeit (Zacher und Keefe 2008). Internationale Regeln, Netzwerke aus Fachleuten, und immer mehr finanzielle Ressourcen für globale Gesundheitspolitik gelten zudem als Beleg für die Diagnose, dass Gesundheit als universelles Gut anerkannt und Gesundheitspolitik Teil der globalen Vergemeinschaftung seien (Youde 2018).

Doch gerade an diesem Politikfeld lässt sich zugleich zeigen, wie das Globale zu einem Begriff wird, der nicht universell-integrierend ist, sondern als Merkmal für periphere Regionen in armen aber auch in reichen Ländern reserviert wird. Ich konzentriere mich dabei auf die Vereinigten Staaten von Amerika (USA), ein Kernland des Weltgesundheitsdiskurses, und zeige, wie dort marginalisierte Gruppen im eigenen Land auf dreifache Weise dem globalen medizinischen Süden eingemeindet werden. Dies geschieht erstens diskursiv, durch die Definition einheimischer globaler Gesundheitsprobleme als die Probleme derer da unten; zweitens institutionell, durch die räumlich Ausweitung der Zuständigkeit von ehedem auf Entwicklungsländer fokussierten Organisationen auf Bereiche im eigenen Land; und drittens auf der Policy-Ebene durch die Übertragung von Versorgungsmodellen, die bislang primär in Entwicklungsländern eingesetzt wurden, auf die heimischen Peripherien. Der globale Blick der Globalisierer wird hier gleichsam zum kolonialen Blick, der die geographische Distanz zu peripheren Ländern wie Ghana mit der sozialen Distanz zu abgehängten Gesellschaftsgruppen innerhalb der USA verschränkt. Aus dieser Sicht ist eine internationale wie nationale Zweiklassenmedizin die logische Konsequenz. Für die breitere Debatte um Global Governance stellt sich angesichts dieser Beobachtung die Frage, ob und wann Weltregieren wirklich nur auf Ungleichheiten reagiert, oder sie nicht auch naturalisiert. 
Im Folgenden diskutiere ich zunächst Vorstellungen von Globalität in den IB. Anschließend schlage ich unter Rückgriff auf die Sozialgeographie vor, Globalisierung als Ungleichheitsheuristik und die Konstruktion post-nationaler Hierarchien zu begreifen. Schließlich skizziere am Beispiel Weltgesundheitspolitik die transnationale Konstruktion eines globalen medizinischen Südens in den USA.

\section{Das Globale in der IB-Theorie}

In den 90er Jahren des vergangenen Jahrhunderts war die Debatte über internationale Institutionen stark mit einer begrifflichen Verschiebung befasst: weg vom internationalen Regieren und hin zum globalen Regieren. Diese Verschiebung gilt weithin als Ausdruck dafür, dass nicht mehr nur Staaten und zwischen-staatliche Institutionen, sondern auch nichtstaatliche Akteure wie Nichtregierungsorganisationen, Unternehmensverbände oder Stiftungen am Projekt Weltregieren teilnehmen (vgl. Dingwerth/Pattberg 2006; Reinicke und Deng 2000). Die gesellschaftliche Triebkraft dieser politischen Neuordnung wurde in der zunehmenden Entgrenzung - Globalisierung - der Gesellschaft verortet, die von Handel über Reisebewegungen bis hin zu kulturellen und normativen Verflechtungen reicht (Beisheim et al. 1999).

Globalisierung wird in der Debatte ums Weltregieren also liberal interpretiert: als Vernetzung von Individuen auf der Grundlage von Werten wie Gleichheit, Demokratie und Marktwirtschaft (vgl. de Wilde et al. 2019). Darin kommt eine spezifische Raumvorstellung des Globalen, wie sie generell in der IB-Theorie vorherrscht, zum Ausdruck. Die globale Gesellschaft (Albert et al. 2013) oder Gemeinschaft (Bartelson 2009), die durch internationale Institutionen gesteuert wird, ist eine Gemeinschaft der prinzipiell gleichwertigen Individuen (Bartelson 2010). Während manche, nicht-liberale Staaten durchaus stratifikatorische Ordnungsprinzipien aufweisen mögen, ist Ungleichheit kein explizites Ordnungsprinzip der globalen Gesellschaft (Zürn et al. 2013). . Faktische Ungleichheit mag aus den Wirtschaftsverhältnissen zwar resultieren und ist gegebenenfalls korrekturbedürftig. Doch zumindest an der Oberfläche ist sie nicht durch esymbolische Ungleichheit zementiert.

Dazu trägt die Symbolik des Globalen bei heißt also hier erdumfassend und egalitär (Bartelson 2009; 2010). National-territoriale Grenzen werden tendenziell irrelevant, denn wir bewegen uns im „supra-territorialen“ (Bartelson 2010: 221, unter Rückgriff auf Scholte 2000) globalen Dorf. 
Damit einher gehen Werte und Ordnungsvorstellungen mit universellem Anspruch, die nicht primär von Nationalstaaten, sondern zunehmend von supranationalen Bürokratien (Barnett/Finnemore 2004), transnationalen Nichtregierungsorganisationen (Boli/Thomas 1999) und Berufsverbänden (Fourcade 2006; Seabrooke/Henriksen 2017) verbreitet werden. Deren Aktivitäten fließen in Vorstellungen eines postnationalen Gemeinwohls ein, die für Global Governance konstitutiv sind (Zürn 2018: 7, 25-36).

Freilich lassen sich konkrete Politikinhalte, wie sie von globalen Institutionen hervorgebracht werden, immer auf ihre Verteilungswirkung oder auch diskriminierenden Charakter befragen (vgl. Enloe 2014; Vitalis 2015); die Existenz von GewinnerInnen und VerlierInnen globaler Politik ist ja gerade ein Antrieb für die politische Soziologie der Global Governance (de Wilde et al. 2019). Davon getrennt gilt es jedoch zu problematisieren, ob die räumlichen Vorstellungen von Supraterritorialität und erdumfassender Integration, wie sie in den IB vorausgesetzt werden, sich wirklich mit den Ordnungsprinzipien der Global Governance decken. Hierzu lohnt sich ein Blick in die Sozialgeographie.

\section{Räumliche Differenzierung jenseits des Nationalstaates}

In der Wirtschaftsgeographie und der Stadtsoziologie wird Globalisierung längst nicht mehr als schlichte Überwindung des Raums oder als das Zeitalter der Supraterritorialität erforscht. Vielmehr betonen Wirtschaftsgeographen wie etwa Neil Brenner, dass durch Globalisierungsprozesse neue Organisationsprinzipien entstanden sind, die auch neue Hierarchien zwischen Räumen jenseits des Nationalstaates erzeugen. Beispielsweise habe die europäische Wirtschaftspolitik sich seit den 1970er Jahren nicht nur vom Keynesianischen Wohlfahrtsstaat abgewandt. Sie habe darüber hinaus auch neue, postnationale Hierarchien zwischen Wirtschaftsräumen hervorgebracht, etwa zwischen urbanen Wachstumszentren und dem abgehängten Hinterland (Brenner 2004). Ähnlich beschreibt Saskia Sassen das Aufkommen von untereinander vernetzten globalen Städten (Sassen 1991), die teils losgelöst von nationalem Territorium organisiert sind, oder identifiziert Michael Goldman den weltweiten Diskurs über erstklassige world cities (Goldman 2011).

Solche wirtschaftsgeographischen Analysen zeigen, wie bestimmte Räume - Städte, Grenzregionen, Sonderwirtschaftszonen - mit besonderen Ressourcen, Regulierungen oder auch Images versehen werden. Die so entstehende räumliche Schichtung ist kulturell 
sanktioniert und fließt, so zeigt insbesondere die Stadtsoziologie, in die unterschiedliche gesellschaftliche Bewertung von Räumen ein. Wenn beispielsweise bestimmte Stadtgebiete mit bestimmten Rassenstereotypen, diskreditierten Verhaltensweisen oder sozialen Problemtypen assoziiert werden, verschränken sich symbolische und räumliche Hierarchisierungen. Schlimmstenfalls bedeuten bestimmte Verortungen von Menschen, etwa als gefährliche und unhygienische BewohnerInnen von Randbezirken europäischer Metropolen, dass deren Schlechterstellung, da räumlich konnotiert, gleichsam natürlich erscheint. Die Klassifikation von Räumen legitimiert damit auch auf subtile Weise die Ungleichbehandlung von Mitmenschen (van Gent/Jaffe 2017).

Auch in globalen Institutionen und sozialen Bewegungen ist die Ausgestaltung von räumlichen Ordnungsbegriffen ein hochpolitischer Prozess. Beispiele hierfür sind Kontroversen über Begriffe wie Entwicklungs- versus Industrieländer oder die Dritte Welt, oder auch die Konstruktion einer westlichen Wertegemeinschaft als zivilisierter Teil der Erde (Dados/Connell 2012; Jackson 2006). Derlei Einteilungen haben Konsequenzen für die unterschiedliche Behandlung von Nationalstaaten durch internationale Institutionen, etwa wenn ärmere Länder von Freihandelsregeln ausgenommen oder für sie gesonderte Klimaschutzziele ausgehandelt werden (Dingwerth et al. 2019). Jenseits dieser Differenzierung zwischen Nationalstaaten (vgl. Viola 2013) lässt sich jedoch, so meine These, mittlerweile zunehmend eine postnationale Form der räumliche Differenzierung beobachten. Das Aidland (Mosse 2013) der Entwicklungspolitik wird zunehmend als transnationaler Raum aufgefasst, so dass die Peripherien von Entwicklungsländern in den globalen Süden eingemeindet werden. Dies zeigt ein Blick auf die globale Gesundheitspolitik der USA, wo sich Vorstellungen von der Ungleichwertigkeit des globalen Südens mit der Exotisierung inländischer Peripherien verschränken.

\section{Das globale Land der Armen in der US Gesundheitspolitik}

„We have global health here, too.” So lautete die Antwort einer angehenden Ärztin, die mir vom Global Health Programm ihrer Klinik in Kalifornien berichtete. ${ }^{2}$ Ich hatte nach den

\footnotetext{
2 Dieser Abschnitt fußt auf diversen empirischen Materialien. Ich habe 2018 und 2019 mehr als 40 Interviews mit US-MedizinerInnen und Gesundheitsfachkräften, die in Global Health Projekten arbeiten, geführt, unter anderem in Albuquerque, Oakland, Chicago, und telefonisch mit Boston, Washington, und Houston. Auch die teilnehmende Beobachtung der Jahreskonferenz 2019 des Consortium of Universitites for Global Health, der Community Health Worker Konferenz in Wenatchee 2019, und des Jahrestreffens 2018 des Social Medicine Consortium fließen in die Analyse ein. Schließlich habe ich Webseiten von US-Universitäten und die medizinische Fachliteratur über Global Health Studiengänge in den USA ausgewertet (vgl. hierzu auch Hanrieder 2019).
} 
Aktivitäten dieses Programms gefragt, und sie nannte mir zunächst Partnerschaften mit einigen Kliniken in Afrika und Asien - um dann, nach kurzem Stocken, weitere Aktivitäten mit benachteiligten Patienten vor Ort zu nennen. Denn, Sie wissen schon, globale Gesundheit gibt es hier auch.

Einerseits ist diese Antwort nicht überraschend. Die USA gelten als wichtiger Ursprungsort des Global Health Diskurses. Das Aufkommen der globalen Begrifflichkeit ist eng verknüpft mit dem Einzug internationaler Gesundheitsbedrohungen in den nationalen Sicherheitsdiskurs der USA, der in den 2000er Jahren stattfand (Weir/Mykhalovskiy 2010). Die amerikanische Gesundheit sollte also zunehmend auch in Übersee und in tropischen Pandemieherden geschützt werden (Rushton 2011). Nationale Interessenpolitik und die Vorstellung von einem globalen Gesundheitsdorf, das ,durch Ansteckung vereint ${ }^{\text {“3 }}$ sei, gehen bei dieser Deutung von globaler Gesundheit Hand in Hand.

Andererseits ist diese Antwort durchaus überraschend. Denn, trotz aller Rede von weltweiten Ansteckungsgefahren und Gesundheitsbedrohungen durch Globalisierung (Lee 2003), wird das Einsatzgebiet globaler Gesundheitspolitik überwiegend außerhalb von Industrieländern verortet. Globale Gesundheit findet in armen Ländern statt und dient einer minimalen Gesundheitsversorgung beziehungsweise Pandemieeindämmung in entfernten Weltregionen (Janes/Corbett 2009; Packard 2016). Sie ist institutionalisiert in internationalen Hilfsprogrammen, Entwicklungsagenturen und in Forschungspartnerschaften zwischen Universitäten in Industrieländern und Standorten im globalen Süden (Crane 2013).

Wenn also hier in Kalifornien globale Gesundheit stattfindet, um was für einen Ort handelt es sich dann? Ein genauerer Blick auf die als global markierten Gesundheitsaktivitäten in den USA zeigt, dass dieses hier keine universelle Verbreitung globaler Politiken (oder auch Bedrohungen) etwa im Sinne eines globalen Gesundheitsdorfs meint. Es geht vielmehr um einen Sozialraum, der sich als globaler medizinischer Süden der USA bezeichnen lässt: Der Hinterhof des US-Gesundheitssystems, der von schlecht oder nicht versicherten, ethnisch Fremden oder anderweitig ausgegrenzten Personen bevölkert wird. Die Konstruktion dieses globalen medizinischen Südens lässt sich an drei Dynamiken festmachen: erstens an der der diskursiven Markierung des heimischen Hinterlands als global, zweitens an der zunehmenden

\footnotetext{
${ }^{3}$ So der Untertitel einer Studie von Zacher und Keefe (2008) zur internationale Kooperation beim Seuchenschutz.
} 
Hinwendung internationaler Gesundheitsorganisationen zu den Problemen dieser Sozialräume und drittens an der Übertragung von Politikmodellen, die noch im 20. Jahrhundert als Drittweltmedizin galten, auf die als global markierten Gegenden der USA.

Die aufkommende Bezeichnung von heimischen Gebieten als global lässt sich besonders deutlich an US-Universitäten nachvollziehen, wo im letzten Jahrzehnt massiv in den Ausbau von Global Health Programmen investiert wurde (Merson 2014). Diese Programme bestehen nicht nur aus Forschungs- und Ausbildungsabkommen mit Partneruniversitäten im globalen Süden (Crane 2013). Zunehmend integrieren die Universitäten auch Einsatzgebiete innerhalb der USA in ihre als globale Gesundheit deklarierten Programme in Forschung und Ausbildung. So zeigt eine Durchsicht der Praktikumsangebote in den Global Health Studienprogrammen der führenden US-Medizinuniversitäten, dass zwanzig von 42 Programmen globale Praxiserfahrungen im eigenen Land anbieten (Hanrieder 2019: 307). Die Spezifizierung der hier ausgewiesenen heimischen Einsatzgebiete bietet Aufschluss über die Art von Raum, der in den USA als global gilt: Es handelt sich hier zum einen um sozioökonomische Kategorien wie die Gruppe der Unterversorgten und von Ressourcenarmut geprägte Gegenden (Macfarlane et al. 2008). Zum anderen sind die Adressaten globaler heimischer Programme ethnisch-kulturelle Gruppen wie Stämme, MigrantIinnen, und sogenannte multikulturelle Kontexte sowie die BewohnerInnen von Grenzregionen. Als Ausbildungsorte gelten derlei Gebiete als besonders geeignet, um globale medizinische Tugenden wie Kultursensibilität und den Umgang mit Fremden sowie die Fähigkeit zur Minimalversorgung unter Bedingungen der Mittelknappheit zu erlernen (vgl. Hanrieder 2019).

Eine zweite Form der Globalisierung der heimischen Peripherie vollzieht sich mit der Ausdehnung des Tätigkeitsraums ehedem internationaler, das heißt auf Einsatzgebiete außerhalb der USA spezialisierter, Gesundheitsorganisationen. So hat sich in letzter Zeit eine Reihe von Organisationen, die bislang vor allem Gesundheitsarbeit außerhalb der USA leistete, neue Einsatzgebiete in den USA erschlossen. Dazu zählt beispielsweise die Nichtregierungsorganisation Partners in Health (PIH), gegründet 1987, die sich mit ihrem Einsatz in Haiti und Ruanda einen Namen gemacht hat. PIH lancierte im Jahr 2009 ein Programm in der indigenen Navajo Nation, das mittlerweile zum eigenständigen Schwesterprogramm wurde und sich für primäre Gesundheitsversorgung inklusive 
Ernährungssicherheit engagiert. ${ }^{4}$ Auch die philanthropische Einrichtung der Sondergesandten für Gesundheit der Vereinten Nationen hat ihre Aktivitäten jüngst auf die USA ausgedehnt, wo sie den Einsatz von primären GesundheitsarbeiterInnen (community health workers) unterstützt. ${ }^{5}$ Und selbst die Weltgesundheitsorganisation (WHO), die gemeinhin eher als Weisungsempfängerin ihrer Geberländer wie insbesondere der USA gilt, verkündete Ende 2018 eine globale Wende: Ihr Generaldirektor Tedros Ghebreyesus kündigte an, zukünftig auch verstärkt auf gesundheitspolitische Probleme wohlhabender Länder Einfluss nehmen zu wollen (Horton 2019).

Drittens schließlich hat in den USA im letzten Jahrzehnt ein Politikmodell an Popularität gewonnen, das bislang als Modell für arme Länder beziehungsweise die Dritte Welt galt: das oben bereits genannte Modell der community health workers (CHW). Dies ist ein Sammelbegriff für Gesundheitspersonal, das üblicherweise keinen höheren Bildungsabschluss hat und Gesundheitsarbeit jenseits medizinischer Eingriffe beziehungsweise außerhalb von Praxen und Krankenhäusern leistet. Hierzu zählen Haus- und Schulbesuche, Aufklärungsarbeit, breitere soziale Hilfestellungen rund um Wohnraum, Nahrung, Aufenthaltsstatus oder Arbeit, manchmal aber auch einfache diagnostische Maßnahmen oder das eigenständige Verabreichen von Impfstoffen (Perry et al. 2014).

In der Weltgesundheitspolitik sind CHWs vor allem als Ikonen der Primary Health Care Bewegung der später 1970er und frühen 1980er Jahre bekannt. Mit dieser Bewegung setzte sich die WHO für ein alternatives Gesundheitsmodell in Entwicklungsländern ein, das weniger auf krankenhausbasierte Versorgung und stattdessen mehr auf sozial verankerte und präventive Dienstleistungen setzte - allen voran erbracht durch CHWs. Sie gelten daher in UNO-Kreisen noch weithin als Drittweltmedizin (Medcalf/Nunes 2018; Perry et al. 2014).

In jüngsten Jahren jedoch findet dieses Politikmodell in den USA immer größere Aufmerksamkeit und auch öffentliche Legitimität. CHWs werden in immer mehr Studien und Pilotprojekten als alternative Fachkräfte erprobt, sind als eigene Berufsgruppe im Arbeitsministerium geführt, und wurden im Affordable Care Act der Obama-Regierung als wertvolle Ergänzung des Gesundheitssystems explizit erwähnt - was eine ganze Welle an

\footnotetext{
${ }^{4}$ Siehe https://www.copeprogram.org/aboutus; 27.08.2019.

5 Vgl. http://www.healthenvoy.org/essential-role-of-community-health-workers-in-the-us-new-report/;

27.08.2019.
} 
neuen (Pilot-)Projekten sowie Initiativen zur Zertifizierung der CHW-Tätigkeit in verschiedenen Staaten beflügelte (Kangovi/Asch 2018).

Bei der laufenden Suche nach CHW-Innovationen in den USA ist hervorzuheben, dass diese Maßnahmen explizit für periphere Gebiete beziehungsweise unterversorgte US-Patienten skaliert werden. Sie richten sich also an den globalen medizinischen Süden im eigenen Land (Singh/Chokshi 2013). Darüber hinaus erlangen Versorgungsmodelle aus dem globalen Süden prominenten Vorbildcharakter, und werden gar zum Ort der Entdeckung des CHW-Modells stilisiert. Zahlreiche Berichte in den Mainstream-Medien fokussieren auf Erzählungen über Initiativen, die von heimgekehrten Internationalisten entwickelt wurden, etwa das New Yorker CHW-Projekt City Health Works, dessen GründerInnen durch ihre Auslandserfahrungen in Indien und Afrika zum CHW-Modell gebracht worden seien (Kenen 2017; Loewenson/Simpson 2017). Auch PIH wird in einem Bestseller als der Harvard Business Press als leuchtendes Beispiel von reverse innovation gefeiert, also als eine Organisation, die neue Modelle für die USA in fernen Ländern entwickelt.

Die Ironie an dieser Entdeckungserzählung ist freilich, dass CHW in den USA schon seit Jahrzehnten am Rande des Gesundheitssystems - in karitativen Projekten, durch Freiwilligenarbeit oder sogenannten community health centers tätig sind. Gerade die Arbeit der CHW in indigenen Reservaten, formalisiert Ende der 1960er, gilt als ältestes offizielles CHWProgramm der USA, das durch PIH allenfalls unterstützt, jedoch nicht eingeführt wurde (KalmFreeman 2009). Dass der aktuelle Mainstreamdiskurs dazu neigt, diese heimischen Vorläufer zu übersehen, beziehungsweise das Rad mithilfe der international mobilen Global Health Elite neu $\mathrm{zu}$ erfinden, führt geradezu zu einer doppelten Marginalisierung der heimischen Gesundheitsperipherie: Einerseits wird sie als Fall für Entwicklungsmedizin dem globalen medizinischen Süden eingemeindet, andererseits werden bestehende und bislang kaum beachtete Formen der (Selbst-)Hilfe am Rande der Gesellschaft weithin ignoriert.

\section{Fazit}

Global Governance wird häufig als Prozess der Verbreitung von Normen und Politiken mit universellem Geltungsanspruch verstanden - ein Prozess, in dem erst einmal alle Individuen gleich sind, und bei dem nur unglückliche Verteilungsprozesse manche Gruppen schlechterstellen. Demgegenüber weist ein Blick auf die Verräumlichung von Global 
Governance darauf hin, dass die Konstruktion von Globalität auch mit symbolischen Grenzziehungen einhergeht.

Am Beispiel der Weltgesundheitspolitik habe ich gezeigt, wie transnationale Diskurse und Institutionen zur Konstruktion einer gleichsam globalen Peripherie in einem Industrieland beitragen: dem globalen medizinischen Süden in den USA. Zwar sind die Ausgrenzung und dramatische medizinische Unterversorgung breiter Bevölkerungsgruppen in diesem Land kein Novum und wurden nicht erst durch die symbolische Eingemeindung in den globalen Süden erzeugt. Doch aus der Geographie und Kultursoziologie ist wohlbekannt, dass die symbolische Grenzziehung die materielle Grenzziehung mit ermöglicht und verfestigt (van gent/Jaffe 2017; Lamont/Molnár 2002). Ein Rückgriff auf Global Health Symbolik und Instrumente der Entwicklungsmedizin zur Addressierung heimischer Ungleichheit mag zwar dem Ziel dienen, diese Ungleichheit zu bekämpfen. Die damit einhergehende symbolische Extraterritorialisierung ganzer Bevölkerungsgruppen trägt aber auch zur institutionellen und symbolischen Verfestigung einer post-nationalen Zweiklassengesellschaft sowie zur Verfremdung und Exotisierung der eigenen NachbarInnen bei (Koptiuch 1991).

Schließlich ist zu betonen, dass die US-Gesundheitspolitik nur ein Fall der Neugestaltung sozialer Räume durch Institutionen der Global Governance ist. Im Bereich Humanitarsmus beispielsweise ist zu beobachten, dass Nichtregierungsorganisationen wie Ärzte ohne Grenzen längst auch Gesundheitskrisen in Wohlfahrtsstaaten addressieren, beispielsweise in Frankreich (Hanrieder/Galesne 2019). Auch sie schaffen neue Raumvorstellungen jenseits des Nationalstaates. Im Bereich der Entwicklungspolitik ist festzustellen, dass viele Staaten ihre Ausgaben für inländische Flüchtlingshilfe als Entwicklungshilfe verbuchen, und damit auch die den Sozialraum der Entwicklung neu vermessen. Solche räumlichen Ausdifferenzierungen des Weltregierens unterstreichen den Bedarf, die politische Soziologie der Global Governance um eine geographische Perspektive zu erweitern. 
Literaturverzeichnis

Albert, Mathias/Buzan, Barry/Zürn, Michael 2013: Introduction: Differentiation Theory and International Relations, in: Albert, Mathias/Buzan, Barry/Zürn,Michael (Hrsg.): Bringing Sociology to Internationale Relations. World Politics as Differentiation Theory, Cambridge, 125 .

Barnett, Michael/Finnemore, Martha 2004: Rules for the World. International Organizations in Global Politics, Ithaca, NY.

Bartelson, Jens 2009: Visions of World Community, Cambridge.

Bartelson, Jens 2010: The Social Construction of Globality, in: International Political Sociology 4: 3, 219-235. 
Beisheim, Marianne/Dreher, Sabine/Gregor, Walter/Zangl, Bernhard/Zürn, Michael (Hrsg.) 1999: Im Zeitalter der Globalisierung? Thesen und Daten zur gesellschaftlichen und politischen Denationalisierung, Baden-Baden.

Boli, John/Thomas, George M. (Hrsg.) 1999: Constructing World Culture: International Nongovernmental Organizations Since 1875, Stanford, CA.

Brenner, Neil 2004: New State Spaces. Urban Governance and the Rescaling of Statehood, New York, NY.

Crane, Johanna T. 2013: Scrambling for Africa. AIDS, Expertise, and the Rise of American Global Health Science, Ithaca, NY.

Dados, Nour/Connell, Raewyn 2012: The Global South, in: Contexts 11: 1, 12-13.

de Wilde, Pieter/Koopmans, Ruud/Merkel, Wolfgang/Strijbis, Oliver/Zürn, Michael (Hrsg.) 2019: The Struggle over Borders. A Political Sociology of Cosmopolitanism and Communitarianism, Cambridge.

Dingwerth, Klaus/Eckl, Julian/Herr, Simon/Weinhardt, Clara 2019: Varieties of Differential Treatment in World Politics. A Historical Look at International Health and Environmental Governance, (Konferenzpapier der SPSA Annual Conference \& Dreiländertagung ETH Zürich), Zürich. 14.-16. Februar 2019.

Dingwerth, Klaus/Pattberg, Philipp 2006: Global Governance as a Perspective on World Politics, in: Global Governance 12: 2, 185-203.

Enloe, Cynthia 2014: Bananas, Beaches and Bases: Making Feminist Sense of International Politics, 2. Auflage, Berkeley, CA.

Fourcade, Marion 2006: The Construction of a Global Profession. The Transnationalization of Economics, in: American Journal of Sociology 112: 1, 145-194. 
Hanrieder, Tine 2019: How Do Professions Globalize? Lessons from the Global South in US Medical Education. International Political Sociology 13: 3, 296-314.

Horton, Richard 2019: Offline. WHO Powers up in 2019, in: The Lancet 393: 10166, 14.

Jackson, Patrick Thaddeus 2006: Civilizing the Enemy. German Reconstruction and the Invention of the West, Ann Arbor, MI.

Janes, Craig R./Corbett, Kitty K. 2009: Anthropology and Global Health, in: Annual Review of Anthropology 38, 167-183.

Kalm-Freeman, Leah 2009: The Community Health Representative Program. Early Voices and Program History 1968-1990 (Promotionsschrift an der Johns Hopkins University), Baltimore, MD.

Kangovi, Shreya/Asch, David A. 2018: The Community Health Worker Boom, in: NEJM Catalyst (August 29), in: https://catalyst.nejm.org/community-health-workers-boom; 26.10.2018.

Kenen, Joanne 2017: New York Borrows a Health Care Idea from Africa. When it Comes to Stretching Resources, America Has Some Things to Learn, in: Politico, 25.10.2017, in: https://www.politico.com/agenda/story/2017/10/25/primary-care-cost-saving-communication$000555 ; 10.12 .2018$.

Koptiuch, Kristin 1991: Third-Worlding at Home, in: Social Text 28, 87-99.

Lamont, Michèle/Molnár, Virág 2002: The Study of Boundaries in the Social Sciences. Annual Review of Sociology 28, 167-195.

Lee, Kelley (Hg.) 2003: Health Impacts of Globalization. Towards Global Governance, Houndmills. 
Loewenson, Rene/Simpson, Sarah 2017: Experiences of Social Power and Participation in Local Health Systems, Volume 1, Key Features of the Short Case Studies, in: Training and Research Support Centre.

Macfarlane, Sarah B./Jacobs, Marian/Kaaya, Ephata E. 2008: In the Name of Global Health. Trends in Academic Institutions, in: Journal of Public Health Policy 29: 4, 383-401.

Medcalf, Alexander/Nunes, João 2018: Visualising Primary Health Care. World Health Organization Representations of Community Health Workers, 1970-89, in: Medical History 62: $4,401-424$.

Merson, Michael H. 2014: University Engagement in Global Health, in: New England Journal of Medicine 370, 1676-1678.

Mosse, David (Hg.) 2013: Adventures in Aidland. The Anthropology of Professionals in International Development, New York, NY.

Packard, Randall 2016: A History of Global Health. Interventions into the Lives of Other Peoples, Baltimore, MD.

Perry, Henry B./Zulliger, Rose/Rogers, Michael M. 2014: Community Health Workers in Low, Middle-, and High-Income Countries. An Overview of Their History, Recent Evolution, and Current Effectiveness, in: Annual Review of Public Health 35, 300-421.

Reinicke, Wolfgang H./Deng, Francis M. 2000: Critical Choices. The United Nations, Networks, and the Future of Global Governance, Washington, DC.

Rushton, Simon 2011: Global Health Security. Security for Whom? Security from What?, in: Political Studies 59: 4, 779-796.

Sassen, Saskia 1991: The Global City, New York, NY.

Scholte, Jan Aart 2000: Globalization. A Critical Introduction, Houndmills. 
Seabrooke, Leonard/Henriksen, Lasse F. (Hrsg.) 2017: Professional Networks in Transnational Governance, Cambridge.

Singh, Prabhjot/Chokshi, Dave A. 2013: Community Health Workers. A Local Solution to a Global Problem, in: New England Journal of Medicine 369: 10, 894-896.

van Gent, Wouter/Jaffe, Rivke 2017: Normalizing Urban Inequality. Cinematic Imaginaries of Difference in Postcolonial Amsterdam, in: Social \& Cultural Geography 18: 4, 553-572, DOI: 10.1080/14649365.2016.1197303.

Viola, Lora Anne 2013: Stratificatory Differentiation as a Constitutive Principle of the International System, in: Albert, Mathias/Buzan, Barry/Zürn, Michael (Hrsg.): Bringing Sociology to International Relations. World Politics as Differentiation Theory, Cambridge, $112-131$

Vitalis, Robert 2015: White World Order, Black Power Politics. The Birth of American International Relations, Ithaca, NY.

Weir, Lorna/Mykhalovskiy, Eric 2010: Global Public Health Vigilance. Creating a World on Alert, New York, NY.

Youde, Jeremy 2018: Global Health Governance in International Society, Oxford.

Zacher, Mark W./Keefe, Tanja J. 2008: The Politics of Global Health Governance: United by Contagion, New York, NY.

Zürn, Michael 2018: A Theory of Global Governance. Authority, Legitimacy, and Contestation, Oxford.

Zürn, Michael/Buzan, Barry/Albert, Mathias (2013): Conclusion. Differentiation Theory and World Politics, in: Albert, Mathias/Buzan, Barry/Zürn, Michael (Hrsg.): Bringing Sociology to International Relations. World Politics as Differentiation Theory, Cambridge, 228-245. 